Vol. 38 (1988) [11-17]

\title{
ON THE INTEGRAL COHOMOLOGY OF THE SEVEN-CONNECTIVE COVER OF BO
}

\author{
Tze Beng Ng
}

\begin{abstract}
Let $B O, B S O$ and BSpin be the classifying spaces for the infinite orthogonal, infinite special orthogonal and infinite spinor groups respectively. It is well known that their integral cohomology rings have torsion only of order 2 . In this paper we present an elementary proof that for the 7-connective cover of $B O, B O\langle 8\rangle$, the integral cohomology ring $H^{*}(B O(8) ; Z)$ too has torsion only of order 2 . The method follows that of Borel and Hirzebruch and a result of Wu concerning the Steenrod reduced mod $p$ operation for an odd prime $p$ on the Pontrjagin classes.
\end{abstract}

\section{INTRODUCTION}

Recently I wished to know whether the integral cohomology of $B O\langle 8\rangle$, the 7 connective cover of $B O$, the classifying space for the infinite orthogonal group, has any element of order $\geqslant 4$. The purpose of this note is to answer this in the negative, more specifically, I prove

TheOREM 1.1. The torsion elements of $H^{*}(B O\langle 8\rangle ; \mathrm{Z})$ are of order 2 .

Recall that $B O\langle 8\rangle$ fibres over BSpin with fibre $K(Z, 3)$, the Eilenberg-MacLane space of type $(Z, 3)$. To prove Theorem 1.1 we need the structure of $H^{*}\left(B O\langle 8\rangle ; Z_{2}\right)$.

\section{COHOMOLOGY of $B O\langle 8\rangle$}

In [4] I defined a system of generators for $H^{*}\left(B S O ; Z_{2}\right)$ as follows.

$$
H^{*}\left(B S O ; \mathbf{Z}_{2}\right) \cong \mathbf{Z}_{2}\left[v_{i} \mid i \geqslant 2\right]
$$

where

$$
v_{i}=\left\{\begin{array}{l}
S q^{2^{r}} \ldots S q^{2^{2}} S q^{2} w_{4} \text { if } i=2^{r+1}+2, r \geqslant 0 \\
S q^{2^{j}\left(2^{i+1}+1\right)} \ldots S q^{2^{t+1}+1} S q^{2^{t}} \ldots S q^{2} w_{4} \text { if } i=2^{i+j+2}+2^{j+1}+1, t \geqslant 0, j \geqslant 0 \\
S q^{2^{j}(2+1)} S q^{2^{j-1}(2+1)} \ldots S q^{2+1} w_{4} \text { if } i=2^{j+2}+2^{j+1}+1, j \geqslant 0 \\
S q^{2^{r-1}} \ldots S q^{2} S q^{1} w_{2} \text { if } i=2^{4}+1, r \geqslant 0 \\
w_{i} \text { otherwise. }
\end{array}\right.
$$

Then a Leray-Serre spectral sequence argument for the fibration $B O\langle 8\rangle \rightarrow \mathrm{BSpin}$ gives:

Received 3 September 1987

Copyright Clearance Centre, Inc. Serial-fee code: 0004-9729/88 \$A2.00+0.00. 
ThEOREM 2.1. $H^{*}\left(B O\langle 8\rangle ; Z_{2}\right) \cong Z_{2}\left[v_{i} ; \alpha(i-1)>2\right]$, where $\alpha(j)=$ number of 1 's in the dyadic expansion of $j$.

According to [4] we have

TheOREM 2.2 .

$$
\begin{aligned}
S q^{1} v_{2^{j}+2} & =v_{2^{j}+2+1} \text { if } j \geqslant 2 \\
S q^{1} v_{2^{j}+2^{k}+1} & =\left(v_{2^{j-1}+2^{k-1}+1}\right)^{2} \text { for } j \geqslant k>1 \\
S q^{1} v_{2^{j}+2+1} & =0, j \geqslant 1 \\
S q^{1} v_{4} & =v_{5}+v_{2} v_{3} \\
S q^{1} v_{2} & =v_{3} .
\end{aligned}
$$

3. The $\mathrm{SQ}^{1}-$ Cономоlogy of $H^{*}\left(B O\langle 8\rangle ; \mathrm{Z}_{2}\right)$

Let $k$ be a field. Let $M=\sum_{k=0}^{\infty} M_{i}$ be a graded vector-space of finite type over a field $k$, that is the dimension of $M_{i}$ is finite for each $i$. Recall that the Poincare Series of $M, P_{k}(M, t)$, is defined by

$$
P_{k}(M, t)=\sum_{i \geqslant 0} \operatorname{dim}\left(M_{i}\right) t^{i}
$$

When $k$ is the field $Z_{p}$, where $p$ is a prime, we write $P_{p}(M, t)$ for $P_{k}(M, t)$ and for the rational field we write $P_{0}(M, t)$ for $P_{\mathbf{Q}}(M, t)$.

By Thomas [5] we have

TheOREM, 3.1.

$$
H^{*}(\mathrm{BSpin} ; \mathbf{Q}) \cong \mathbf{Q}\left[Q_{1}, \ldots, Q_{i}, \ldots\right]
$$

where $Q_{i}$ are generators in dimension $4 i$.

Since the fibre of $B O\langle 8\rangle \rightarrow$ BSpin is $K(Z, 3)$ and since $H^{*}(K(Z, 3) ; Q) \cong$ $H^{*}\left(S^{3} ; Q\right)$, a Leray-Serre spectral squence argument for rational cohomology gives us

TheOREM 3.2.

$$
H^{*}(B O\langle 8\rangle ; \mathbf{Q}) \cong \mathbf{Q}\left[Q_{2}, \ldots, Q_{j}, \ldots\right]
$$

Hence

Corollary 3.3 .

$$
P_{0}\left(H^{*}(B O\langle 8\rangle ; Q), t\right)=\prod_{j \geqslant 2}\left(1-t^{4 j}\right)^{-1}=U(t)
$$

In order to commute the $\mathrm{Sq}^{1}$ cohomology of $H^{*}\left(B O\langle 8\rangle ; \mathrm{Z}_{2}\right)$ we shall need the following 
Theonem 3.4. (Borel-Hirzebruch [1])

$$
\begin{aligned}
P_{2}\left(H\left(H^{*}\left(B S O ; Z_{2}\right), S q^{1}\right), t\right) & =P_{0}\left(H^{*}(B S O ; Q), t\right) \\
& =\prod_{j \geqslant 1}\left(1-t^{4 j}\right)^{-1}=Q(t) .
\end{aligned}
$$

Now we shall rename the $v_{i}$ for $\alpha(i-1) \leqslant 2$. Define $R_{0}$ to be $v_{2}$. For integers $i \geqslant$ $j$ define $R(i, j)$ to be $v_{2^{i}+2^{j}+1}$. For Example, $R(0,0)=v_{3}, R(1,0)=v_{4}, R(1,1)=v_{5}$ and $R(i, i)=v_{2^{i+1}+1} i \geqslant 0$. As in [4] we define $L(k, j), k \geqslant j \geqslant 0$ to be the polynomial subalgebra over $Z_{2}$ generated by $R(r, s), k>r \geqslant s \geqslant 0$ or $k=r$ and $j \geqslant s$ together with $R_{0}$. Let the ideal generated by $L(k, j)$ be denoted by $\bar{L}(k, j)$.

Define $M(k, j), k \geqslant j \geqslant 0$, by

$$
M(k, j)=H^{*}\left(B S O ; \mathbf{Z}_{2}\right) / \bar{L}(k, j) .
$$

Define $M(0)=H^{*}\left(B S O ; \mathrm{Z}_{2}\right) /\left(v_{2}\right)$. By Theorem $2.2 \mathrm{Sq}^{1}(L(k, j)) \subseteq L(k, j)$ for $k \geqslant$ $j \geqslant 1$. Since $\mathrm{Sq}^{1}: H^{*}\left(B S O ; \mathrm{Z}_{2}\right) \rightarrow H^{*}\left(B S O ; \mathrm{Z}_{2}\right)$ is a derivation and $\mathrm{Sq}^{1} \mathrm{Sq}^{1}=0$, the cohomology $H\left(H^{*}\left(B S O ; \mathrm{Z}_{2}\right), \mathrm{Sq}^{1}\right)=\mathrm{Ker} \mathrm{Sq}{ }^{1} / \mathrm{Im} \mathrm{Sq}{ }^{1}$ is a graded vector space over $Z_{2}$. By abuse of notation we shall write $d$ for

$$
\left.\mathrm{Sq}^{1}\right|_{L(k, j)} k \geqslant j \geqslant 1 \text { or } \mathrm{Sq}^{1}: M(k, j) \rightarrow M(k, j) .
$$

Then $H^{*}\left(B O\langle 8\rangle ; Z_{2}\right)=\underset{\operatorname{Lim}}{\rightarrow} M(k, j)$. Now

$$
\left(H^{*}\left(B S O ; \mathrm{Z}_{2}\right), \mathrm{Sq}^{1}\right)=\left(\mathrm{Z}_{2}\left[v_{2}, v_{3}, v_{4}, v_{5}\right], d\right) \otimes(\dot{M}(1,1), d) .
$$

Thus $P_{2}\left(H^{*}\left(B S O ; \mathrm{Z}_{2}\right), \mathrm{Sq}^{1}\right)=\left(1-t^{4}\right)^{-1}\left(1-t^{8}\right)^{-1} P_{2}(M(1,1), d)$ where we denote $P_{2}\left(H\left(H^{*}\left(B S O ; \mathrm{Z}_{2}\right), \mathrm{Sq}^{1}\right), t\right), P_{2}(H(M(1,1), d), t)$ by $P_{2}\left(H^{*}\left(B S O ; \mathrm{Z}_{2}\right), S^{1}\right)$ and $P_{2}(M(1,1), d)$, respectively.

Now for $(j, k), j \geqslant k>1$, it follows from Theorem 2.2 that we have the following exact sequence of chain complexes

$$
0 \rightarrow \sum^{2^{j}+2^{k}+1} M(j, k-1) \stackrel{\cdot R(j, k)}{\longrightarrow} M(j, k-1) \rightarrow M(j, k) \rightarrow 0
$$

where $\left(\sum^{2^{j}+2^{k}+1} M(j, k-1)\right)_{r}=(M(j, k-1))_{r-2^{j-2^{k}-1}}$ and $\cdot R(j, k)$ means multiplication by $R(j, k)$. This short exact sequence induces the long exact sequence

$$
\begin{aligned}
\cdots \rightarrow H^{i}\left(\sum^{2^{j}+2^{k}+1} M(j, k-1)\right) & \rightarrow H^{i}(M(j, k-1)) \rightarrow H^{i}(M(j, k)) \\
& \rightarrow H^{i+1}\left(\sum^{2^{j}+2^{k}+1} M(j, k-1)\right) \rightarrow \ldots
\end{aligned}
$$


which is equivalent to

$$
\begin{aligned}
\ldots & \rightarrow H^{i-2^{j}-2^{k}-1}(M(j, k-1)) \rightarrow H^{i}(M(j, k-1)) \\
& \rightarrow H^{i}(M(j, k)) \rightarrow H^{i-2^{j}-2^{k}}(M(j, k-1)) \rightarrow \ldots
\end{aligned}
$$

3.7 Claim: $H^{2 r+1}(M(j, k-1))=0$ for $j \geqslant k, k \geqslant 2$. Assuming this claim, we then have from (3.6) the following short exact sequence

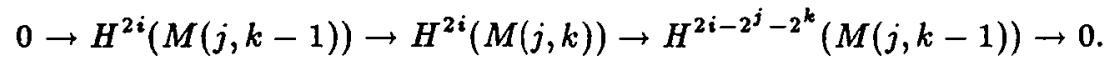

Therefore $H^{2 i}(M(j, k))=H^{2 i}(M(j, k-1)) \oplus H^{2 i-2^{j}-2^{k}}(M(j, k-1))$. Thus we have

$$
P_{2}(M(j, k), d)=\left(1+t^{2^{j}+2^{k}}\right) P_{2}(M(j, k-1), d)
$$

for $k>1$. Now for $k \geqslant 2$,

$$
(M(k-1, k-1), d) \cong(M(k, 1), d) \otimes\left(Z_{2}[R(k, 0), R(k, 1)], d\right)
$$

Since $d(R(k, 0))=R(k, 1)$, for $k \geqslant 2$

$$
P_{2}(M(k-1, k-1), d)=\left(1-t^{2\left(2^{k}+2\right)}\right)^{-1} P_{2}(M(k, 1), d)
$$

Thus for $k \geqslant 2$

$$
P_{2}(M(k, 1), d)=\left(1-t^{2^{k+1}+2^{2}}\right) P_{2}(M(k-1, k-1), d)
$$

\subsection{Proof of Claim 3.7:}

$$
\begin{aligned}
P_{2}(M(1,1), d) & =P_{2}\left(H^{*}\left(B S O ; \mathrm{Z}_{2}\right), \mathrm{Sq}^{1}\right)\left(1-t^{4}\right)\left(1-t^{8}\right) \\
& =Q(t) \cdot\left(1-t^{4}\right)\left(1-t^{8}\right)
\end{aligned}
$$

Thus $H^{2 i+1}(M(1,1), d)=0 . H^{2 i+1}(M(j, k-1))=0$ for $j>k, k \geqslant 2$ is proved by induction on $(j, k-1)$. It $k=2$, then it follows from $(3.9)_{j}$ and induction hypothesis. If $k>2$ then if follows from the exact sequence (3.6) and the induction hypothesis.

Now from $(3.7)_{(j, k)}$ we have

$$
P_{2}(M(j, k), d)=\left(1+t^{2^{j}+2^{k}}\right)\left(1+t^{2^{j}+2^{k-1}}\right) \ldots\left(1+t^{2^{j}+2^{2}}\right) P_{2}(M(j, 1), d) .
$$


This together with $(3.9)_{j}$ gives us:

$$
\begin{aligned}
& P_{2}(M(j, k), d)=\prod_{k \geqslant r \geqslant 2}\left(1+t^{2^{j}+2^{r}}\right) \prod_{j-1 \geqslant s \geqslant r \geqslant 2}\left(1+t^{2^{2}+2^{r}}\right) \\
& \prod_{j \geqslant r \geqslant 2}\left(1-t^{2^{r+1}+2^{2}}\right) P_{2}(M(1,1), d) \\
& \prod_{\substack{j-1 \geqslant 0 \geqslant r \geqslant 2 \\
s=j, k \geqslant r \geqslant 2}}\left(1+t^{2^{2}+2^{r}}\right) \prod_{j \geqslant r \geqslant 2}\left(1-t^{2^{r+1}+2^{2}}\right) Q(t) \cdot\left(1-t^{4}\right)\left(1-t^{8}\right) \\
& =\left(1-t^{4}\right) Q(t) \prod_{\substack{j-1 \geqslant 0>r \geqslant 2 \\
\theta=j, k \geqslant r \geqslant 2}}\left(1+t^{2^{s}+2^{r}}\right) \prod_{j \geqslant r \geqslant 2}\left(1-t^{2^{r+1}+2^{2}}\right) \\
& \cdot\left(1-t^{2^{j+1}}\right)
\end{aligned}
$$

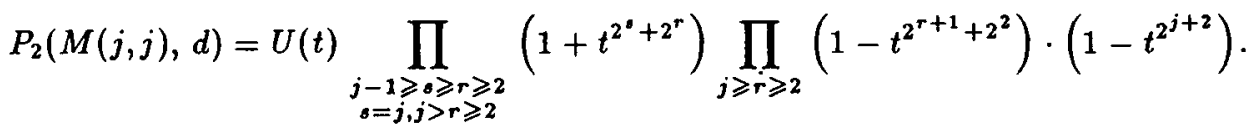

Since

$$
\prod_{s>r \geqslant 2}\left(1+t^{2^{2}+2^{r}}\right) \prod_{r \geqslant 2}\left(1-t^{2^{r+1}+2^{2}}\right)=1
$$

we see that

$$
P_{2}(\stackrel{\operatorname{Lim}}{\longrightarrow} M(k, j), d)=\left(1-t^{4}\right) Q(t)=\prod_{j \geqslant 2}\left(1-t^{4 j}\right)^{-1}
$$

Therefore we have

THEOREM 3.11 .

$$
P_{2}\left(H^{*}\left(B O\langle 8\rangle ; \mathbf{Z}_{2}\right), S q^{1}\right)=P_{0}\left(H^{*}(B O\langle 8\rangle ; \mathbf{Q})\right)
$$

From Theorem 3.11 and [1] we have

THEOREM 3.12. The 2-primary component of $H^{*}(B O\langle 8\rangle ; Z)$ has order 2 only.

4. THE MOD $p$ COHOMOLOGY OF $B O\langle 8\rangle$ FOR ODD PRIME $p$

Consider the mod $p$ Leray-Serre cohomology spectral sequence for $K(\mathbb{Z}, 3) \longrightarrow$ $\mathrm{BO}\langle 8\rangle \longrightarrow \mathrm{BSpin}$

By Cartan [3] $H^{*}\left(K(Z, 3), \mathbf{Z}_{p}\right)$ is an anti-commutative algebra generated by

$$
\left\{\mathcal{P}^{p^{k}} \mathcal{P}^{p^{k-1}} \ldots \mathcal{P}^{p} \mathcal{P}^{1} \iota_{3}, \beta \mathcal{P}^{p^{k}} \mathcal{P}^{p^{k-1}} \ldots \mathcal{P}^{1} \iota_{3} \mid k \geqslant 0\right\}
$$


where $\mathcal{P}^{p^{i}}$ are the Steenrod reduced $\bmod p$ operations and $\beta$ is the Bockstein operation associated with the exact sequence $0 \rightarrow Z_{p} \rightarrow Z_{p^{2}} \rightarrow Z_{p} \rightarrow 0$ and $\iota_{3}$ is the fundamental class of $K(\mathbf{Z}, 3)$.

Now by Thomas $[5], H^{*}\left(B S p i n ; Z_{p}\right) \cong Z_{p}\left[P_{1}, P_{2}, \ldots\right]$ where $P_{i} \in H^{4 i}\left(B S p i n ; Z_{p}\right)$ is the $\bmod p$ Pontrjagin class.

Let $\left\{E_{r}, d_{r}\right\}$ be the $\bmod p$ Leray-Serre spectral squence for $B O\langle 8\rangle \rightarrow$ BSpin . Then $E_{2} \cong H^{*}\left(\mathrm{BSpin} ; \mathrm{Z}_{p}\right) \otimes H^{*}\left(K(3, \mathrm{Z}), \mathrm{Z}_{p}\right)$. Then:

$$
\begin{aligned}
& d_{4} \iota_{3}=\frac{1}{2} P_{1} \\
& d_{2\left(p^{k+1}+1\right)}\left(\mathcal{P}^{k} \ldots \mathcal{P}^{k-1} \mathcal{P}^{p} \mathcal{P}^{l} \iota_{3}\right)=\frac{1}{2} \mathcal{P}^{k} \ldots \mathcal{P}^{1} P_{1} \\
& d_{2\left(p^{k+1}+1\right)+1}\left(\beta \mathcal{P}^{p^{k}} \ldots \mathcal{P}^{1} \iota_{3}\right)=0 .
\end{aligned}
$$

By Wu [6] $\mathcal{P}^{p^{k}} \ldots \mathcal{P}^{p} \mathcal{P}^{1} P_{1}=P_{\left(p^{k+1}+1\right) / 2}$ modulo decomposables. We shall now define a system of generators for $H^{*}\left(\mathrm{BSpin} ; \mathbf{Z}_{p}\right)$. Let

$$
\begin{aligned}
& v_{0}=P_{1}, \\
& v_{k}=\mathcal{P}^{p^{k-1}} \ldots \mathcal{P}^{P} \mathcal{P}^{1} P_{1} \text { for } k \geqslant 1 .
\end{aligned}
$$

Then $\operatorname{dim} v_{k}=2\left(p^{k}+1\right)$. Thus $\left\{v_{0}, v_{1}, v_{2}, \ldots\right\}$ together with $P_{i}$ for $i \neq \frac{p^{k}+1}{2} k \geqslant 0$ generates $H^{*}\left(\mathrm{BSpin} ; \mathrm{Z}_{p}\right)$.

By the usual spectral sequence argument we have:

TheoREM 4.1. $H^{*}\left(B O\langle 8\rangle ; \mathrm{Z}_{p}\right) \cong H^{*}\left(\mathrm{BSpin} ; \mathrm{Z}_{\mathrm{p}}\right) / L \otimes \Lambda$ where $\Lambda$ is the subalgebra generated by $\left\{\beta \mathcal{P}^{p^{k}} \ldots \mathcal{P}^{1} \iota_{3}, k \geqslant 0\right\}$ and $L$ is the ideal generated by $\left\{v_{0}, v_{1}, \ldots, v_{k}, \ldots\right\}$.

Let $R_{k}=H^{*}\left(\mathrm{BSpin} ; \mathbf{Z}_{p}\right) /\left(v_{0}, v_{1}, \ldots v_{k}\right)$. For $k \geqslant 0$ we have the following exact sequence

$$
0 \longrightarrow \sum^{2\left(p^{k+1}+1\right)} R_{k} \stackrel{v_{k+1}}{\longrightarrow} R_{k} \longrightarrow R_{k+1} \longrightarrow 0 .
$$

Thus $P_{p}\left(R_{k+1}, t\right)+t^{2\left(p^{k+1}+1\right)} P_{p}\left(R_{k}, t\right)=P_{p}\left(R_{k}, t\right)$. That is $P_{p}\left(R_{k+1}, t\right)$ $=\left(1-t^{2\left(p^{k+1}+1\right)}\right) P_{p}\left(R_{k}, t\right)$. Hence we have

THEOREM 4.2.

$$
\begin{aligned}
P_{p}\left(R_{k}, t\right) & =\prod_{j \geqslant 1}^{k}\left(1-t^{2\left(p^{j}+1\right)}\right) P_{0}\left(R_{0}, t\right) \\
& =\prod_{j \geqslant 1}^{k}\left(1-t^{2\left(p^{j}+1\right)}\right) \prod_{j \geqslant 2}\left(1-t^{4 j}\right)^{-1} .
\end{aligned}
$$


It follows from Theorem 4.1 and Theorem 4.2 that

$$
P_{p}\left(H^{*}\left(\text { BSpin; } Z_{p}\right) / L, t\right)=\prod_{j \geqslant 1}\left(1-t^{2\left(p^{j}+1\right)}\right) \prod_{j \geqslant 2}\left(1-t^{4 j}\right)^{-1} .
$$

Now $P_{p}(\Lambda, t)=\prod_{k \geqslant 1}\left(1-t^{2\left(p^{k}+1\right)}\right)^{-1}$. Therefore

$$
\begin{aligned}
P_{p}\left(H^{*}\left(B O\langle 8\rangle ; Z_{p}\right), t\right) & =P_{p}\left(H^{*}\left(B S p i n ; Z_{p}\right) / L, t\right) P_{p}(\Lambda, t) \\
& =\prod_{j \geqslant 2}\left(1-t^{4 j}\right)^{-1}=P_{0}\left(H^{*}(B O\langle 8\rangle ; \mathbf{Q}), t\right) .
\end{aligned}
$$

Thus we prove:

TheOREM 4.3. $H^{*}(B O\langle 8\rangle ; \mathrm{Z})$ has no $p$-torsion for odd prime $p$.

Theorem 1.1 now follows from Theorem 3.12 and Theorem 4.3.

\section{REFERENCES}

[1] A. Borel and F. Hirzebruch, 'Characteristic classes and homogeneous spaces II', Amer. J. Math. 81 (1959), 315-382.

[2] A. Borel, Lecture Notes in Mathematics 36, in (Springer Verlag, 1967).

[3] H. Cartan, 'Sur les groupes d'Eilenberg-MacLane $H(\pi, n), 1,11$, , Proc. Nat. Acad. Sci., U.S.A. vol 60 (1954), 467-471, 704-707:

[4] Tze-Beng $\mathrm{Ng}$, 'A note on the mod 2 cohomology of $B \hat{S} O_{n}<16>$ ', Canad. J. Math. 37 (1985), 893-907.

[5] E. Thomas, 'On the cohomology groups of the classifying space for the stable spinor group', Bol. Soc. Mat. Mexicana 7 (1962), 57-69.

[6] W.T. Wu, 'On Pontrjagin classes II', Acta. Math. Sinica 4 (1954), 171-199.

Department of Mathematics National University of Singapore

Singapore 